\title{
Mitigation of Voltage Sags and Voltage Swells by Dynamic Voltage Restorer
}

\author{
${ }^{1}$ D. Rajasekaran and ${ }^{2}$ Subhransu Sekhar Dash \\ ${ }^{1}$ Department of Electrical and Electronics Engineering, \\ R.M.D. Engineering College, Chennai, 601-206 Tamil Nadu, India \\ ${ }^{2}$ Department of Electrical and Electronics Engineering, SRM University, \\ Chennai, 603-203 Tamil Nadu, India
}

\begin{abstract}
Modern industrial processes are based on a large amount of electronic devices such as programmable logic controllers and adjustable speed drives. Unfortunately, electronic devices are sensitive to disturbances and thus industrial loads become less tolerant to power quality problems such as voltage sags, voltage swells and harmonics. The Dynamic Voltage Restorer (DVR) has become popular as a cost effective solution for the protection of sensitive loads from voltage sags and swells. This study deals with modeling and simulation of a Dynamic Voltage Restore (DVR) for mitigation of voltage sags and voltage swells. The control of the compensation voltages in DVR based on $\mathrm{d}-\mathrm{q}-\mathrm{o}$ algorithm is discussed. The controller in $\mathrm{d}-\mathrm{q}-\mathrm{o}$ coordinates has better performance than conventional controllers. The controlled variables in $\mathrm{d}-\mathrm{q}-\mathrm{o}$ coordinates are then inversely transformed to the original voltages which produced reference voltages to a DVR. The DVR restores constant load voltage and voltage wave form by injecting an appropriate voltage. Effectiveness of proposed technique is investigated through computer simulation by using Matlab/Simulnk software. The simulation results have shown validation of the control system.
\end{abstract}

Key words: Dynamic voltage restorer, voltage sags, voltage swells, power quality, voltage source inverter, d-q-o coordinates, India

\section{INTRODUCTION}

Modern industrial equipments are more sensitive to power quality problems such as voltage sag, voltage swells interruption, harmonic, flickers and impulse transient. Failures due to such disturbances create high impact on production cost (Kantaria and Joshi, 2008). So now-a-days, high quality power is became basic needs of highly automated industries.

Voltage sag is the most sever power quality problem faced by industrial customers. Voltage sag is common reasons for malfunctioning in production plants. Voltage sag is a short term reduction in voltage magnitude. According to IEEE standard 1159, voltage sag is a decrease in RMS voltage between $10-90 \%$ at a power frequency for duration from 0.5 cycles to $1 \mathrm{~min}$ (IEEE Std. 1159,1995 ).

Voltage swell is less severs compare to voltage sag because they are less common in distribution system. The definition of voltage swell quoted: a voltage is in increase in RMS voltage $>110 \%$ at the power frequency for duration from 0.5 cycles to $1 \mathrm{~min}$ (IEEE Std. 1159, 1995). Voltage swell generate due to switching of capacitor bank and switching off large load. The effect of voltage swell is control delay, tripping, overheating and many times destruction in electrical equipments (Li et al., 2006). Power electronics and advanced control technologies have made it possible to mitigate power quality problems and maintain the operation of sensitive loads. There are a number of voltage sag/swell mitigating methods available but the use of custom power service is considered to the most efficient method.

This study introduce basic concept of DVR (Dynamic Voltage Restorer). DVR inject an appropriate voltage magnitude with an appropriate phase angle dynamically. This study deals with modeling and simulation of a Dynamic Voltage Restore (DVR) for mitigation of voltage sags and voltage swells. The control of the compensation voltages in DVR based on d-q-o algorithm is discussed. The controller in $\mathrm{d}-\mathrm{q}-\mathrm{o}$ coordinates has better performance than conventional controllers. The controlled variables in $\mathrm{d}-\mathrm{q}-\mathrm{o}$ coordinates are then inversely transformed to the original voltages which produced reference voltages to a DVR. The DVR restores constant load voltage and voltage wave form by injecting an appropriate voltage (Hingorani and Gyugyi, 1999).

Corresponding Author: D. Rajasekaran, Department of Electrical and Electronics Engineering, R.M.D. Engineering College, Chennai, 601-206 Tamil Nadu, India 
Effectiveness of proposed technique is investigated through computer simulation by using Matlab/Simulnk software. The simulation results have shown validation of the control system.

Dynamic voltage restorer: A dynamic voltage restorer is one of the custom power devices which is used to protect sensitive loads from sag, swell and harmonics or disturbances in the supply voltage. The DVR consists of a voltage source inverter, a switching control scheme, a DC energy storage device, output filter and an injection or coupling transformer is connected in series with the ac system as shown in Fig. 1. DVR uses semi-conductor device to maintain voltage of sensitive load by injecting voltage whose magnitude, phase and frequency can be controlled.

Therefore as shown in Fig. 1, the DVR is located on approach of sensitive loads. If a fault occurs on other lines, DVR inserts series voltage $\mathrm{V}_{\mathrm{dvr}}$ and compensates load voltage to pre fault value. The momentary amplitudes of the three injected phase voltages are controlled such as to eliminate any detrimental effects of a bus fault to the load voltage $\mathrm{V}_{\mathrm{L}}$. This means that any differential voltages caused by transient disturbances in the ac feeder will be compensated by an equivalent voltage generated by the converter and injected on the medium voltage level through the booster transformer. The DVR works independently of the type of fault or any event that happens in the system provided that the whole system remains connected to the supply grid, i.e., the line breaker does not trip.

For most practical cases, a more economical design can be achieved by only compensating the positive and negative sequence components of the voltage disturbance seen at the input of the DVR. This option is reasonable because for a typical distribution bus configuration, the zero sequence part of a disturbance will not pass through the step down transformer because of infinite impedance for this component. The DVR has two modes of operation which are; standby mode and boost mode. In standby mode $\left(\mathrm{V}_{\mathrm{dvr}}=0\right)$, the booster transformer's low voltage winding is shorted through the converter.

No switching of semiconductors occurs in this mode of operation because the individual converter legs are triggered such as to establish a short-circuit path for the transformer connection.

Therefore, only the comparatively low conduction losses of the semiconductors in this current loop contribute to the losses. The DVR will be most of the time in this mode. In boost mode $\left(V_{\text {dv }}>0\right)$, the DVR is injecting a compensation voltage through the booster transformer due to a detection of a supply voltage disturbance (Nielsen et al., 2004).

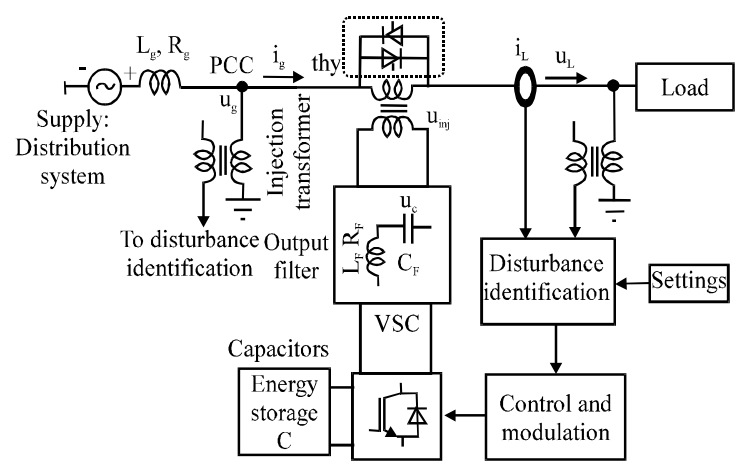

Fig. 1: Single line diagram of dynamic voltage restorer

Design of dynamic voltage restorer: The aim of the control scheme is to maintain constant voltage magnitude at the sensitive load under voltage disturbance condition. The proposed control scheme based on comparison of actual supply voltage and desired load voltage. The error is determined dynamically based on difference between desired and measured value. In the control scheme, the actual voltage is measured and also the desired voltage. These voltages are converted in d-q-o with the parks transformation (Joos et al., 2002).

Control scheme: The basic functions of a controller in a DVR are the detection of voltage sag/swell events in the system; computation of the correcting voltage, generation of trigger pulses to the sinusoidal PWM based DC-AC inverter, correction of any anomalies in the series voltage injection and termination of the trigger pulses when the event has passed.

The controller may also be used to shift the DC-AC inverter into rectifier mode to charge the capacitors in the DC energy link in the absence of voltage sags/swells. In the control scheme, the actual voltage is measured and also the desired voltage. These voltages are converted in dqo with the parks transformation (Joos et al., 2002):

$$
\mathrm{f}_{\mathrm{dqo}}=\mathrm{K}_{\mathrm{s}} \mathrm{f}_{\mathrm{abc}}
$$

Where:

$$
\begin{gathered}
\left(\mathrm{f}_{\mathrm{dq} q}\right)^{\mathrm{T}}=\left(\mathrm{f}_{\mathrm{d}} \mathrm{f}_{\mathrm{q}} \mathrm{f}_{0}\right) \\
\left(\mathrm{f}_{\mathrm{abc}}\right)^{\mathrm{T}}=\left(\mathrm{f}_{\mathrm{a}} \mathrm{f}_{\mathrm{b}} \mathrm{f}_{\mathrm{c}}\right) \\
\mathrm{K}_{\mathrm{s}}=\left(\begin{array}{ccc}
\cos \theta & \cos \left(\theta-\frac{2 \pi}{3}\right) & \cos \left(\theta+\frac{2 \pi}{3}\right) \\
\sin \theta & \sin \left(\theta-\frac{2 \pi}{3}\right) & \sin \left(\theta+\frac{2 \pi}{3}\right) \\
\frac{1}{2} & \frac{1}{2} & \frac{1}{2}
\end{array}\right)
\end{gathered}
$$


It can be shown that for the inverse transformation we have:

$$
\left(\mathrm{K}_{\mathrm{s}}\right)^{-1}=\left(\begin{array}{ccc}
\cos \theta & \sin \theta & 1 \\
\cos \left(\theta-\frac{2 \pi}{3}\right) & \sin \left(\theta-\frac{2 \pi}{3}\right) & 1 \\
\cos \left(\theta+\frac{2 \pi}{3}\right) & \sin \left(\theta+\frac{2 \pi}{3}\right) & 1
\end{array}\right)
$$

The control system employs abc to dqo transformation to dqo voltages. During normal condition and symmetrical condition, the voltage will be constant and d-voltage is unity in p.u. and q-voltage is zero in p.u. but during the abnormal conditions, it varies. After comparison $\mathrm{d}$ and $\mathrm{q}$ voltage with the desired voltage error $\mathrm{d}$ and $\mathrm{q}$ is generated. This error component is converted into abc component using dqo to abc transformation. Software Phase Locked Loop (SPLL) is used to generate unit sinusoidal wave in phase with main voltage. This abc components are given to generate 3-phase pulses using Sine Pulse Width (SPWM) technique. Proposed control technique block is shown in Fig. 2.

\section{RESULTS AND DISCUSSION}

DVR is simulated for voltage sag/swell by using Matlab/Simulnk.

Mitigation of balanced voltage sags: In the simulation model, the voltage sags are generated by switching on impedance to the ground at the Point of Common Coupling (PCC), upstream the DVR. This situation is equivalent to a remote short-circuit fault which results in

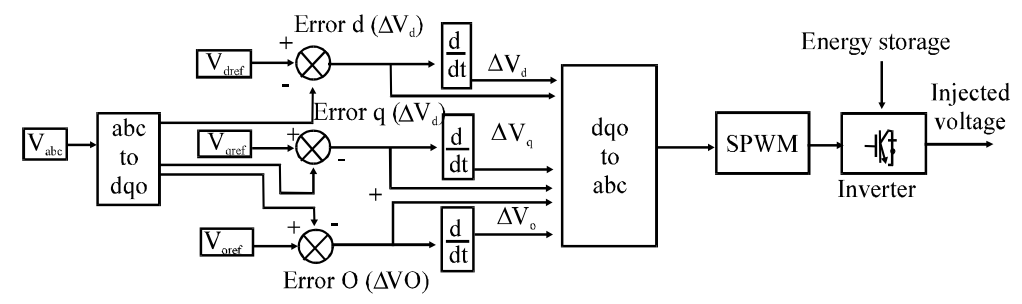

Fig. 2: Control technique block diagram
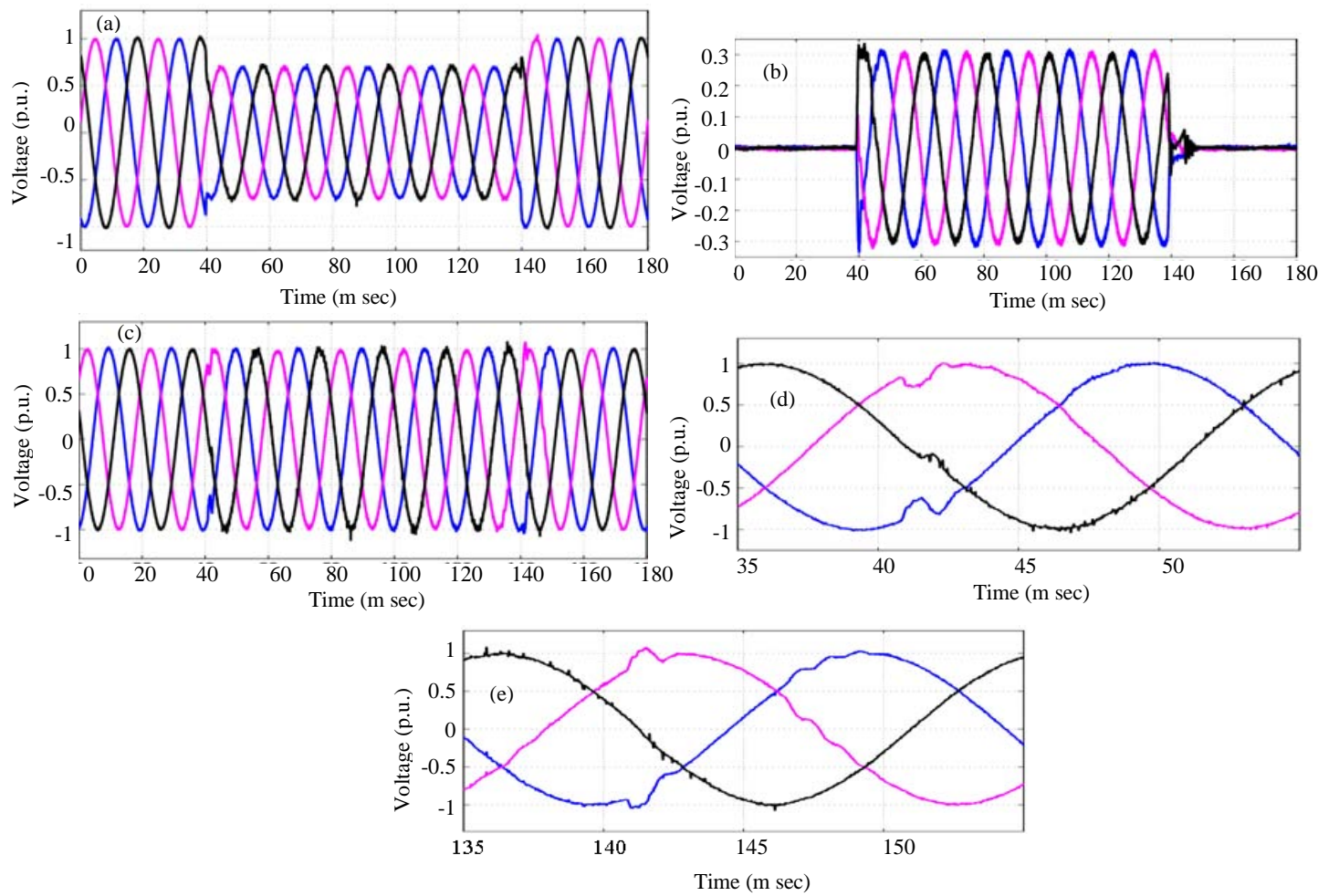

Fig. 3: a) Grid voltage; b) Injected voltage; c) Load voltage; d) Zoom at sag start in load voltage waveform and e) Zoom at sag end in load voltage waveform 

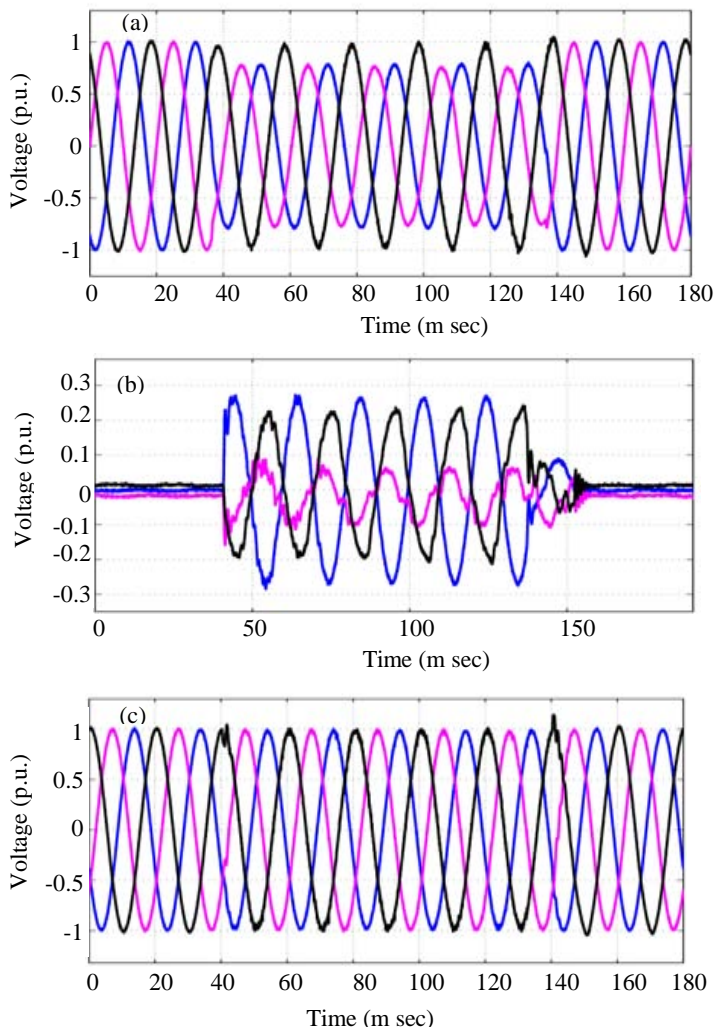

Fig. 4: a) Grid voltage; b) Injected voltage and c) Load voltage

voltage dips with a phase angle jump. The result for simulation of voltage sag is shown in Fig. 3a-c. A $70 \%$ voltage sag is initiated for 5 cycles at the PCC as shown in Fig. 3a. The injected and the load voltage is shown in Fig. 3b, c. A zoom on the load voltage at the sag start is shown in Fig. 3d while a zoom at the sag end is shown in Fig. 3. The results confirm the improved transient performance of the DVR.

Mitigation of unbalanced voltage sags: An unbalanced sag is programmed to generate the grid voltage at the PCC as shown in Fig. 4a. One phase stays at 1 p.u. during the dip and other two phases drop to 0.75 p.u. The injected voltage by the DVR and the restored load voltage is shown in Fig. 4b, c. Also, the error in the magnitude of the load voltage is shown in Fig. 4d. Before the sag, the DVR is in the standby state waiting for the sag detection.

When the positive sequence of the grid voltage drops $<90 \%$, the sag is detected and the DVR starts compensation. It is noted from Fig. $4 \mathrm{c}$ that the compensated load voltage is balanced and the maximum error is $<2 \%$ which indicates the capability of the DVR to cope with unbalanced sags.
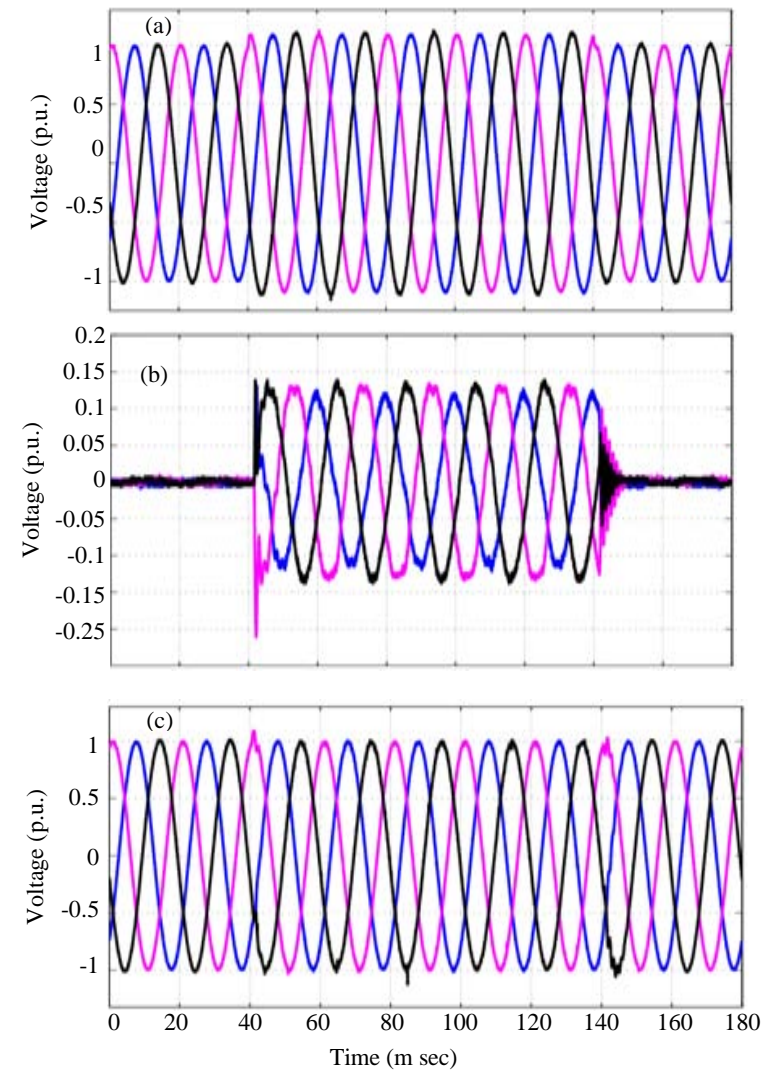

Fig. 5: a) Grid voltage; b) Injected voltage and c) Load voltage

Mitigation of balanced and unbalanced voltage swells: From the control point of view, the DVR should handle voltage swells in the same way it handles voltage sags. In either case, the reference of the injected voltage is stepped and the actual voltage has to track its reference. But it is a different perspective from the energy handling capability. In the case of voltage sags, the DVR delivers an active power to the load but in the case of voltage swells, the DVR may absorb the power from the grid. Since, normally, the DVR is operated at the standby and the required energy is available in its energy storage, the power absorption may be dangerous unless a dissipative element is installed and the power dissipation is controlled. The control of power dissipation in this context is referred to as the online overvoltage protection. Therefore, the capability of the DVR to cope with voltage swells is strongly related to the online overvoltage protection. However in some cases, the voltage swells may not cause an overvoltage at the dc link. The voltage swell characteristics and the loading conditions are the main issues that determine the energy transfer status from the grid to the DVR. Two cases of measured voltage swells are presented here. A $10 \%$ balanced voltage swell 

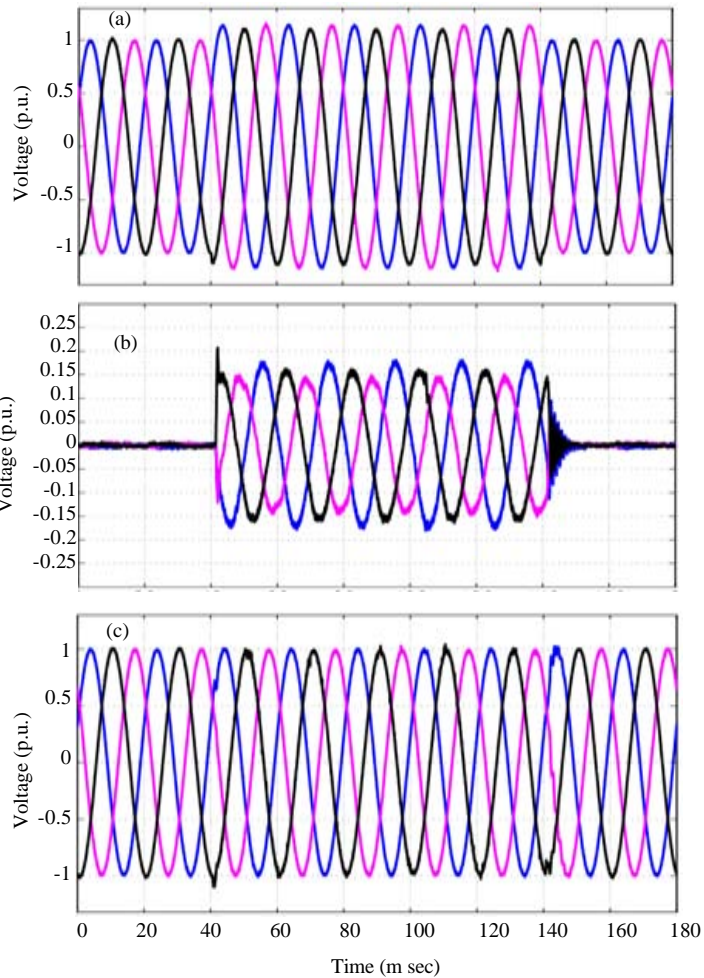

Fig. 6: a) Grid voltage; b) Injected voltage and c) Load voltage

is programmed and measured at the PCC. The grid voltage is shown in Fig. 5a where a swell of $10 \%$ is made for 5 fundamental cycles. The injected voltage and the load voltage is shown in Fig. 5b, c. From Fig. 5a-c, it is noted that the DVR has successfully kept the load voltage at 1 p.u.

Figure 6 shows the case of unbalanced voltage swell where the voltages of the supply are programmed to have $10 \%$ voltage swell. The grid voltage is depicted at Fig. $6 \mathrm{a}$ where a swell of $10 \%$ is made for 5 fundamental cycles. The injected voltage and the load voltage is shown in Fig. 6b, c. From Fig. 6a-c, it is noted that the DVR has successfully kept the load voltage at 1 p.u.

Although, the swell is unbalanced, the DVR can keep the load voltage balanced. During experiments, the ESC was fed by a separate dc source and thus the dc voltage was fixed at $480 \mathrm{~V}$ (0.8 p.u.).
As the measured voltage swells are not relatively large, they have not influenced the dc voltage.

\section{CONCLUSION}

The Dynamic Voltage Restorer (DVR) is a promising and effective device for power quality enhancement due to its quick response and high reliability. The role of a DVR in mitigating the power quality problems in terms of voltage sag, swell is explained. This study has proposed the modeling and simulation of DVR using simulink in Matlab. The very simple abc to dqo based control technique used. Simulation result show the DVR mitigates voltage sags and swells very fast. The conclusion is that the DVR is an effective apparatus to protect sensitive loads from short duration voltage sags and swells.

\section{REFERENCES}

Hingorani, N.G. and L. Gyugyi, 1999. Understanding FACTS: Concepts and Technology of Flexible AC Transmission Systems. 1st Edn., Wiley IEEE Press, New York, USA., SBN-13: 978-0-7803-3455-7, pp: 452.

IEEE Std. 1159, 1995. Recommended practice for monitoring electric power quality. IEEE Standards Coordinating Committee 22 on Power Quality, USA. http://www.apqi.org/file/attachment/2008721/11311 2.pdf.

Joos, S.C., G. Lopes and L.W. Guo, 2002. A nonlinear control method of voltage restorers. IEEE 33rd Ann. Power Electron. Specialists Conf., 1: 88-93.

Kantaria, R. and S.K. Joshi, 2008. A review on power quality problems and solutions. Proceedings of the Power Electronics National Conference, November 2008.

Li, Y., C. Mao, B. Zhang and J. Zeng, 2006. Voltage sag study for a practical industrial distribution network. Proceedings of the International Conference on Power System Technology, Oct. 22-26, Chongqing, pp: 1-4.

Nielsen, J.G., M. Newman, H. Nielsen and F. Blaabjerg, 2004. Control and testing of dynamic voltage restorer (DVR) at medium voltage level. IEEE Trans. Power Electron., 19: 806-813. 